\title{
Design a Multi-Touch Table and Apply to Interior Furniture Allocation
}

\author{
Chien-Hsu Chen, Ken-Hao Nien, and Fong-Gong Wu \\ Department of Industrial Design, National Cheng-Kung University \\ No.1, University Road, Tainan 701, Taiwan \\ chenhsu@mail.ncku.edu.tw, music923@gmail.com, \\ fonggong@mail.ncku.edu.tw
}

\begin{abstract}
This is a study based on the integration of FTIR multi-touch technology with Industrial Design to produce a multi-touch table. An multi-touch system interface is also developed through this study. Furniture allocation is applied as the content to provide users practical operating experience on the multi-touch interface. The process includes FTIR structure related testing, hardware technology and specifications; and the exterior design. The system interface includes image recognition system and multi-touch application, and is developed in FLASH. This study not only uses the easy-to-use characteristics of the multi-touch technology but also integrates PV3D to link the 3D scene with the user interface. This provides a real-time 3D simulation image that the user can view the result of the furniture allocation while controlling the user interface. Observation and interviews are made on the users to evaluate the advantages and related problems of the multi-touch technology for future study and development.
\end{abstract}

Keywords: Multi-Touch, Interior Design.

\section{Introduction}

With the development and popularization of computer, massive digital information has leaded us to enter the age of digitizing. In this age, computing is closely linked with our life. For instance, digital document, music, map, mail etc, and the digital information also have changed the method of paper writing, data saving or information sending. Not only thus it can be seen massive digital information has changed our life, on the other hand it has also brought us a convenient life. And then we could find that keyboard and mouse are the most popular way to interactive with digital information no mater it is simple or not.

But regarding such interactive way, recently, some scholars have proposed different views. Ishii and Ullmer [1] then proposed a new thought in the human-machine interaction-Tangible Bits. They thought that digital information should allow users to grasp or manipulate, but not have to use the keyboard or mouse. For this purpose, they have described three key concepts of Tangible Bits: interactive surface; the coupling of bits with graspable physical objects; and ambient media for background awareness. Its goal is to integrate the digital information into physical environment 
for achieving easy to use with natural operation in order to reduce learning in digital world. Enable the information to be manipulated directly in the intuition way is also called Tangible User Interface.

Besides Tangible User Interface, Mitsubishi Electric Research Laboratories (MERL) have presented their study on the new type of interface [2]. They provided a touch screen that users can directly operate on it. Different from signal touch screen, it has the multi-touch and multi-user characteristics, enriching the operation interface. Before MERL, Rekimoto [3] also presented the related research about multi-touch technology called SmartSkin project. From NYU, Han [4] also presented the multitouch technology called frustrated total internal reflection (FTIR). Although the technologies of multi-touch are different, they have the same purpose to let users directly manipulate virtual information on the screen with their fingers and also bring the possibility of multi-user simultaneously working together.

Thus it can be seen, the computer interface operation is no longer limited in the traditional mouse and keyboard. The new operating mode unceasing was proposed. Both the TUI and multi-touch have the same purpose on a more natural behavior. However, research apply these interactive interface in the tabletop or large-scale display. Because the user does not need to look at the computer screen, operate keyboard and mouse. This has changed the operation behavior of interface and increased the future interface from existence degree of freedom.

Recently also has related research on combining the digital information with our physical environment for providing an intuition interface through different combination. For instance, Park et al. [5] presented a series of future wisdom family equipment in the "Smart home". Without the complex technology and user interface, it can provide awareness of information according to user's demand and take the user to the better life experience. And in some case, as we can see, they combined the digital information with around environment, for instance, furniture, wall, windows or tabletop. And then Sukeda et al. [6] also present the concept of information-accessing furniture. They embed the technical equipment in the table, wall and mirrors providing the different information in different situation. They hope that this can help users more easily to get the information from daily life.

From the related research, the information display and the interaction of human and digital information will not only be through the use of screen, keyboard or mouse. With the development of new interaction interface will enhance those concepts to be come true. Although multi-touch, one of those research, is still at the initial development stage, it is getting popular and expending quickly because of the simple and intuitive operation. So the goal of this study is to integrate the multi-touch technology and design from the point of view of Industrial Design. In order to make it come true, this study describes the integration of multi-touch technology and design. A multitouch table is made and an interface for interior furniture allocation is designed to demonstrate its application and provide different kind of user experience.

\section{Design and Implementation}

At the beginning, we have to choose the multi-touch technology first and understand the related technical equipment. After that, we could integrate it with design process. 
Considering the low-tech, low cost and design implementation, we finally choose the FTIR technology. In the beginning of the design, we tested the related materials and equipments in order to make sure the related size and technology, including the projector position, size, the capturing area of camera and the electric circuit of IR-LED. After several testing, we recorded the related positions between projector, camera, mirror, and the area of image to design a foundation for them (Fig. 1).

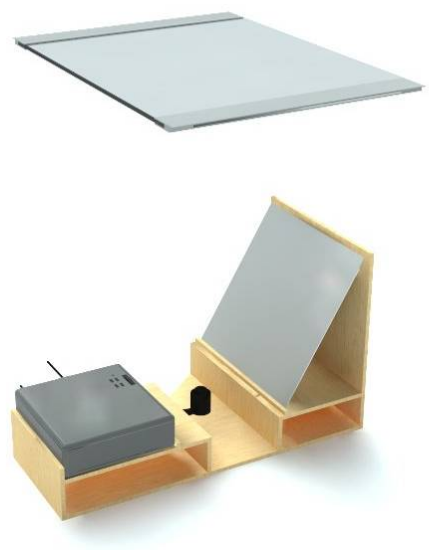

Fig. 1. The design of foundation

Next, we extended foundation design into the table design, and several idea sketches were drawing with the outward appearance concept to have chosen (Fig. 2). Then, we co-manufacture the table with the furniture company to make it.

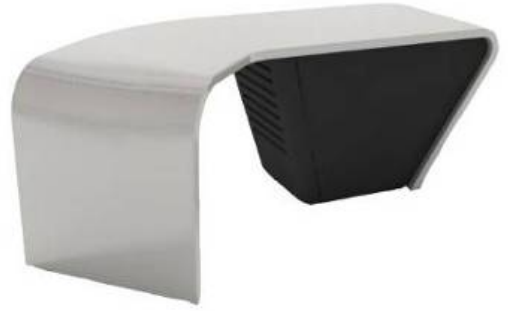

Fig. 2. The outward appearance of table design

On the two sides (right and left) of projecting screen, the electric circuits of IRLED were made. Each side has five groups of infrared LED; there are ten groups on each side. Each group has five infrared LED and adds one $130 \mathrm{ohm}$ resistance, and the distance between two infrared LED is $1.8 \mathrm{~cm}$. we made five infrared LED in a series connection, and then put ten groups in a parallel connection.

During the manufacturing process, some assembling and equipment testing were made for final checking (Fig. 3). In the final stage, color and pattern planning had been done to complete the final work (Fig. 4). 


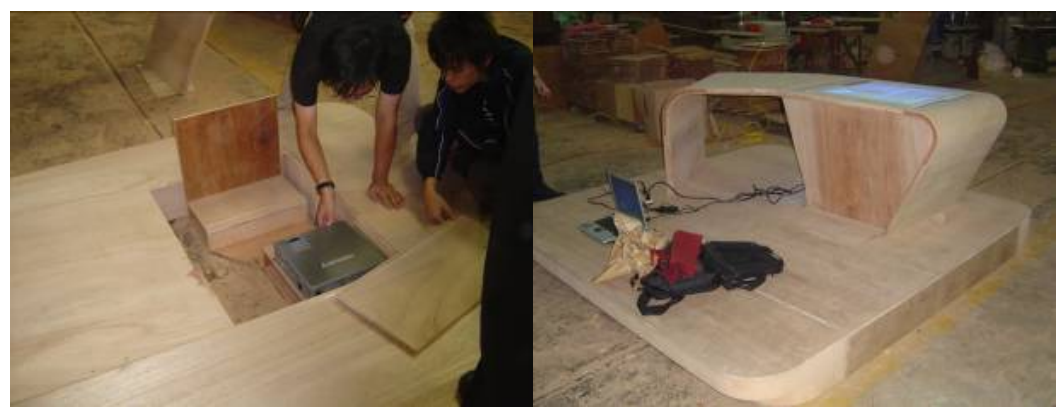

Fig. 3. Assembling and equipment testing

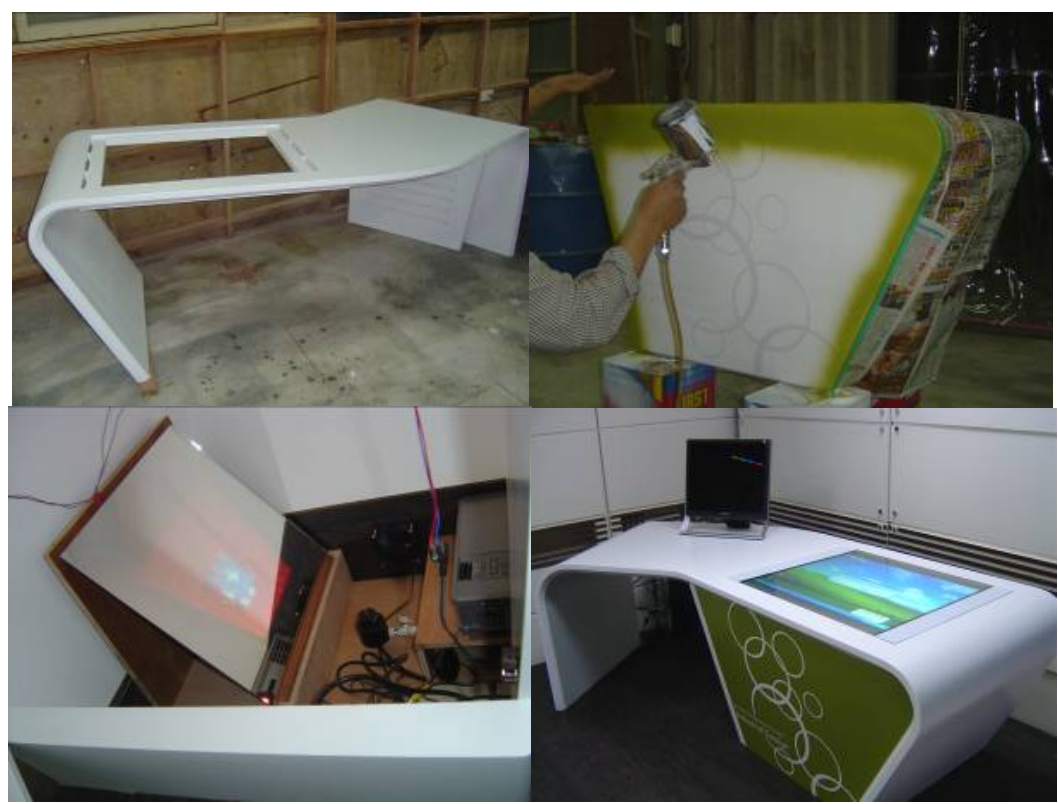

Fig. 4. Final accomplishment

\section{Software System}

After the design is completed, we created a system through FLASH to develop multitouch image recognition system which is called Blob Tracking, and apply to interior furniture allocation.

Blob Tracking is used to trace the white point position when the user touches on the screen, and then sending the points' data to FLASH application. Therefore, we can use those data to define different manipulation meaning. For example, signal point can represent the drag target, and two points can rotate or scale the target. This not only could achieve the characteristics of multi-touch, simultaneously also reduce 
the difficulty of computer programming for industrial designers such that they can concentrate in the interface design application.

\section{Interior Furniture Allocation}

Concerning the interface, we designed an interior furniture allocation for application. Through the characteristics of the multi-touch, users can drag and rotate furniture to complete the allocation from Bird-view. Because of the GUI, the environment of single point, it usually has to manipulate object through other menus. But multi-touch can manipulate the object directly, it is more intuitive and simple, through different definition, it will extend richer manipulations. So in the multi-touch environment, it will decrease the complication of manipulation. And provide users an easy-to-use manipulation.

On the other hand, in the past, it was natural to have only one view to complete the allocation task, after finishing the task, and then have a 3D render. This kind of process not only spends much time but also use only one view to complete the task. It is not easy to image the $3 \mathrm{D}$ space from only one view. So we design a simultaneous $3 \mathrm{D}$ view for the users to see while they are allocating the furniture.

\subsection{Interface}

This study combines FLASH with PV3D to design the interface. PV3D is a set of FLASH 3D technology. It can simulate 3D in FLASH application. Therefore, we use FLASH and PV3D to design the interior furniture allocation interface and simulate the 3D render image. Users can simply drag or rotate to allocate the furniture into ideal interior spatial position. While allocating the furniture into space, user can view a real-time 3D image related to his/her furniture allocation or user can switch to the first person viewpoint to view the result of the furniture allocation and modify the allocation. (Fig. 5)

The furniture of the interface takes the reference of the IKEA product, choosing the classified living room as the system subject. The 3D model is constructed by 3DS Max. In order to reduce loading of system, we must construct the 3D models by lower spot. Besides, we modified all textures through Photoshop in order to get more quality of 3D models.

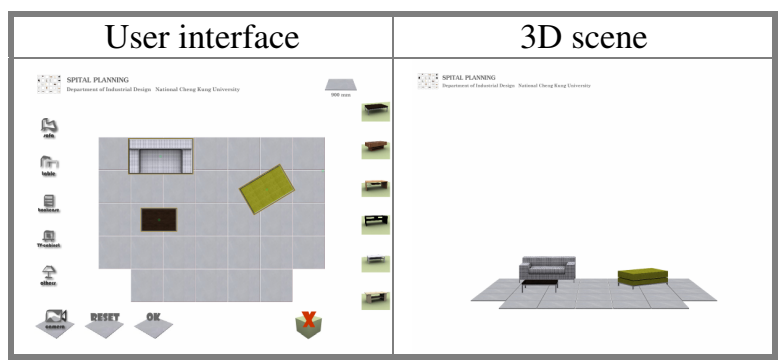

Fig. 5. Interface 


\subsection{Playing Experience}

We invited 20 users to operate the interface. During the operation, the users have to finish the allocation without any limitation (Fig. 6).

The result of the interview and observation are as follows: They think that multitouch is intuitive, convenient and free. The manipulation is easier and faster than the mouse. In the past, using mouse usually has to manipulate objects through RightClick or other icons, but multi-touch can manipulate objects directly, it is easier and faster. So it also can manipulate furniture quickly, allocate their own interior space. And through 3D, it can bring them more real feel of space, including the relative position, relative height and the reality. One can also see the whole scene with different view from camera. So most users think that simultaneous 3D screen help them a lot. Furthermore, some users point that multi-touch allow multi-user, so it is possible for them to discuss the furniture allocation with their family or designer and client have a platform to discuss.

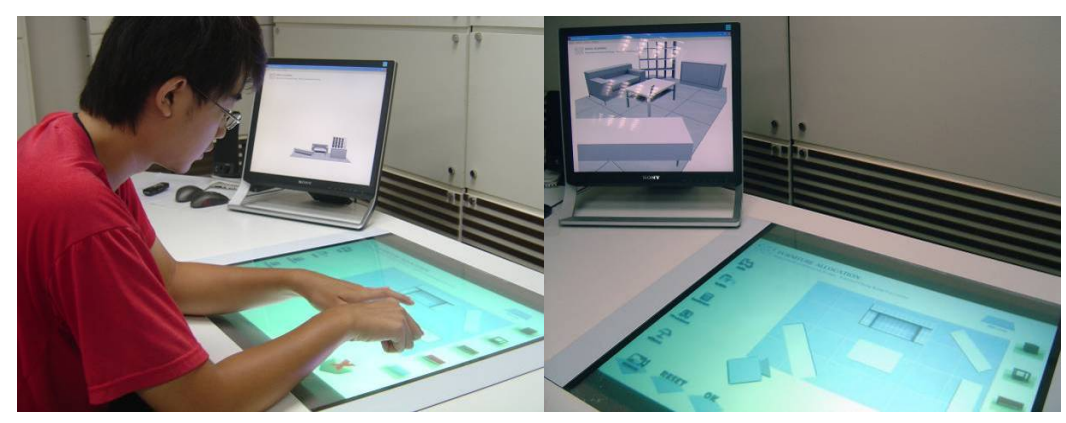

Fig. 6. Playing experience

Besides, some users mentioned that although multi-touch is very intuition and simple to manipulate, but they feel that must be reasonable and not have too complicated gesture. If it were too complicated and need to be remember excessive it would become a bad effect. Also there is another worthy question is about accurate. It is not easy for us to manipulate small objects on the interface because of our fingers have their own limitation. So there are have some shortages about accurate to be improved in the future.

\section{Conclusion}

After this experience of the integration of design, we discovered that there are still trifle place to be improved such as assembling in the manufacturing process. We have realized the difficulty and had the questions to be at after the integrating technology with design. However, in this study, we show the multi-touch technology can be integrated into the traditional furniture design process and provide the implementation possibility for future furniture design. And, a FLASH based multi-touch imagerecognition software is development as the SDK of the multi-touch interface design in 
FLASH environment such that designers can use it to develop different multi-touch applications. In addition, we develop an interior furniture allocation system to demonstrate the design work of multi-touch table.

The results of this study, most users have positive opinion on multi-touch, and also think it will be a new trend in the future. But most users think that there is a main problem when they operated the multi-touch table. The problem is insensitivity. Because of insensitivity, users have to manipulate it with more forces, it will increase the trouble of manipulation imperceptibly. So after this study, we will look for some possible materials and methods to improve the problem, and star to plan a larger multi-touch screen and some other different form to develop at the same time. Looking forward to possible developments in the future.

\section{References}

1. Ishii, H., Ullmer, B.: Tangible bits: towards seamless interfaces between people, bits and atoms. In: Pemberton, S. (ed.) Proceedings of the SIGCHI Conference on Human Factors in Computing Systems, CHI 1997, pp. 234-241. ACM Press, New York (1997)

2. Shen, C.: Multi-User Interface and Interactions on Direct-Touch Horizontal Surfaces: Collaborative Tabletop Research at MERL. In: Proceedings of the First IEEE International Workshop on Horizontal Interactive Human-Computer Systems (2006)

3. Rekimoto, J.: SmartSkin: an infrastructure for freehand manipulation on interactive surfaces. In: Proceedings of the SIGCHI Conference on Human Factors in Computing Systems: Changing Our World, Changing Ourselves. CHI 2002, pp. 113-120. ACM Press, New York (2002)

4. Han, J.Y.: Low-cost multi-touch sensing through frustrated total internal reflection. In: Proceedings of the 18th Annual ACM Symposium on User interface Software and Technology. UIST 2005, pp. 115-118. ACM Press, New York (2005)

5. Park, S., Won, S., Lee, J., Kim, S.: Smart home - digitally engineered domestic life. Personal Ubiquitous Comput. 7(3-4), 189-196 (2003)

6. Sukeda, H., Horry, Y., Maruyama, Y., Hoshino, T.: Information-Accessing Furniture to Make Our Everyday Lives More Comfortable. IEEE Transactions on Consumer 178 Electronics 52(1) (February 2006) 